\title{
Association between SLCO1B1 rs4149056 and tegafur-uracil-induced hepatic dysfunction in breast cancer
}

\author{
Hidenori Kamio', Toshitaka Uchiyama², Hitoshi Kanno², Yoshiko Onoe ${ }^{3}$, Kayoko Saito², \\ Shingo Kameoka ${ }^{1}$, Takako Kamio*,1 \& Takahiro Okamoto ${ }^{1}$ \\ ${ }^{1}$ Department of Surgery II, School of Medicine, Tokyo Women's Medical University, 8-1 Kawada-cho, Shinjuku-ku, Tokyo \\ 162-8666, Japan \\ ${ }^{2}$ Institute of Medical Genetics, School of Medicine, Tokyo Women's Medical University, 8-1 Kawada-cho, Shinjuku-ku, Tokyo \\ 162-8666, Japan \\ ${ }^{3}$ Department of Obstetrics \& Gynecology, School of Medicine, Tokyo Women's Medical University, 8-1 Kawada-cho, Shinjuku-ku, \\ Tokyo 162-8666, Japan \\ *Author for correspondence: kamio.takako@twmu.ac.jp
}

\begin{abstract}
Aim: The aim of this study was to identify pharmacogenomic biomarkers to predict tegafur-uracil (UFT)induced liver dysfunction. Patients \& methods: A total of 68 patients, who were administered UFT, were evaluated using a two-step pharmacogenomics analysis. Results: The first screening revealed the association between five SNPs and UFT-induced hepatic dysfunction. In the second step, SLCO1B1 (rs4149056) was found to be the only SNP associated with UFT treatment-related elevation of aspartate aminotransferase (odds ratio: $C / C$ vs $T / T=7.8, C / T$ vs $T / T=5.7 ; p=0.037$ ) and alanine transaminase (odds ratio: $C / C$ vs $T / T=12.2, C / T$ vs $T / T=4.1 ; p=0.034$ ) levels. Conclusion: The $S L C O 1 B 1$ polymorphisms are possible predictors of UFT treatment-related hepatic dysfunction.
\end{abstract}

First draft submitted: 15 June 2018; Accepted for publication: 4 January 2019; Published online: 8 February 2019

Keywords: breast cancer $\bullet$ hepatic dysfunction $\bullet$ pharmacogenomics $\bullet$ single nucleotide polymorphism $\bullet$ SLCO1B1 - tegafur/uracil

Tegafur-uracil (UFT) is a preparation combining tegafur, a prodrug of fluorouracil (5-FU), and uracil. The UFT is converted by CYP2A6 to an unstable intermediate, 5-hydroxytegafur, which spontaneously breaks down to form 5-FU. More than $80 \%$ of it is metabolized in the liver. The UFT is a drug used widely for breast and other solid cancers such as gastric, rectal and lung cancers [1-3] as postoperative adjuvant therapy because of its low incidence of adverse effects and ease of administration [4-6]. Hepatic dysfunction is one of the serious adverse effects induced by UFT, and approximately $20 \%$ of patients develop elevations of aspartate aminotransferase (AST) and alanine transaminase (ALT) levels [7,8]. Moreover, some cases develop fulminant hepatitis that can be fatal [9].

Previous studies reported that genetic polymorphisms in DPYD and TYMS were associated with severe toxicities, such as diarrhea, mucositis and leucopenia, induced by 5-FU [10]. However, other studies reported that genetic polymorphisms in DPYD and TYMS were not associated with severe toxicity induced by 5-FU [11-13]. To date, no genomic biomarker predicting 5-FU/UFT-induced hepatic dysfunction has been identified [11,14]. Therefore, the present study aimed to identify a predictive biomarker associated with UFT-induced hepatic dysfunction.

A two-step pharmacogenomics analysis was performed to elucidate a SNP that can predict liver dysfunction in UFT therapy. In the first step, 1936 SNPs in 225 genes encoding for drug-metabolizing enzymes and drug transporters were genotyped in 36 patients using the DMET ${ }^{\mathrm{TM}}$ Plus GeneChip microarray, and SNPs significantly associated with grades 1-4 hepatic toxicities were screened. In the second step, five candidate SNPs chosen in the first step were examined in the other 32 patients to verify the associations with elevated AST and ALT levels.

Future Medicine 


\begin{tabular}{|c|c|}
\hline SNP & Sequence \\
\hline \multicolumn{2}{|c|}{ rs3731722 in AOX1: } \\
\hline - Forward & 5'-TCAAGGGTCTGGGAGAGTCG-3' \\
\hline - Reverse & 5'-GCGTTCACGAAAACTTACCACA-3' \\
\hline \multicolumn{2}{|c|}{ rs2762934 in CYP24A1: } \\
\hline - Forward & 5'-TCACAGTAAGCCACACCTTTTA-3' \\
\hline - Reverse & 5'-AGGGCCTGAAACGGTTTGTA-3' \\
\hline \multicolumn{2}{|c|}{ rs1061040 in SLC7A7: } \\
\hline - Forward & 5'-TCTTTGCCTTAGCCCCTTC-3' \\
\hline - Reverse & 5'-AGTCACCTTCATTGTCCCC-3' \\
\hline \multicolumn{2}{|c|}{ rs683369 in SLC22A1: } \\
\hline - Forward & 5'-AGCCCAGGGATACCGAGTT-3' \\
\hline - Reverse & 5'-CGCTTTCTCTCAGCCCACTA-3' \\
\hline \multicolumn{2}{|c|}{ rs4149056 in SLCO1B1: } \\
\hline - Forward & 5'-TCTTCCTTCATCTTCCGCC-3' \\
\hline - Reverse & $5^{\prime}$-TCATCAATGTAAGAAAGCCCC-3' \\
\hline
\end{tabular}

\section{Patients \& methods \\ Patients}

A total of 68 Japanese female breast cancer patients treated with UFT alone or combination therapy of UFT plus endocrine therapy as postoperative adjuvant therapy in our department from 1998 to 2015 were enrolled in this study.

The UFT dose ranged from 150 to $350 \mathrm{mg}$ per body surface area according to the patient's age and clinical status. Toxicity was classified according to the National Cancer Institute Common Toxicity Criteria (NCI-CTC, version 3.0).

\section{Data collection}

To measure AST and ALT levels, venous blood was sampled before administration, at 1, 2 and 3 months after the first administration of UFT, and every 3 months thereafter until UFT discontinuation.

\section{Genotyping}

The DMET ${ }^{T M}$ Plus system (Affymetrix, CA, USA) was used to search for candidate SNPs responsible for UFTinduced hepatic dysfunction. This system can analyze 1936 SNPs in 225 genes involved in drug metabolism, excretion and transport.

In the second step, PCR products flanking the five candidate SNPs were sequenced using Sanger sequencing. The primers used in PCR are listed in Table 1.

\section{Statistical analysis}

In the first step, a related analysis was performed between genotypes and grades 0-4 elevated AST and ALT levels for 36 of 68 patients. The Wilcoxon signed-rank test was used to determine significant differences in dominant and recessive inheritance models, and the Jonckheere-Terpstra trend test was used to determine significant differences in the additive inheritance model. If one of the three p-values was significant, this identified an SNP associated with elevated AST and ALT levels. The SNPs that were significantly associated with elevations of both AST and ALT levels were selected.

In the second step, for 32 of 68 patients, the associations between candidate SNPs chosen in the first step and grades 0-4 elevated AST and ALT levels were confirmed using the analyses described in the first step.

Based on the results of step 1, we assumed that the proportion of the risk population was 30\%, the expression ratio of grade 2-3 in the risk group was $60 \%$, while the expression ratio of grade 2-3 in the non-risk group was $15 \%$. Based on the above setting, it was calculated that 32 cases are required to secure a detection power of $80 \%$ at a significance level of $10 \%$ based on a one-sided Fisher's exact test on a $2 \times 2$ partition table. 
Table 2. Patients' demographics and clinical characteristics.

\begin{tabular}{|c|c|c|}
\hline Demographic or clinical characteristics & Step $1(n=36)$ & Step $2(n=32)$ \\
\hline Age at administration, mean (SD) & $64.3(14.3)$ & $66.5(13.3)$ \\
\hline \multicolumn{3}{|l|}{ Clinical stage: } \\
\hline$-\| \mathrm{A}$ & $14(39 \%)$ & $7(22 \%)$ \\
\hline$-I I B$ & $7(19 \%)$ & $6(19 \%)$ \\
\hline- IIIA & $4(11 \%)$ & $2(6.6 \%)$ \\
\hline$-I I I C$ & $4(11 \%)$ & $4(12.5 \%)$ \\
\hline \multicolumn{3}{|l|}{ Subtype: } \\
\hline$-\mathrm{HR}+/ \mathrm{HER} 2-$ & $12(34 \%)$ & $14(44 \%)$ \\
\hline$-\mathrm{HR}+/ \mathrm{HER} 2+$ & $2(5.6 \%)$ & $3(9.4 \%)$ \\
\hline - HR-/HER2+ & $4(11 \%)$ & $3(9.4 \%)$ \\
\hline$-G 2$ & $1(2.7 \%)$ & $0(0 \%)$ \\
\hline$-\mathrm{G} 3$ & $4(11 \%)$ & $3(9.4 \%)$ \\
\hline$-G 4$ & $0(0 \%)$ & $0(0 \%)$ \\
\hline \multicolumn{3}{|l|}{ Toxicity grade: elevated ALT: } \\
\hline$-\mathrm{GO}$ & $12(33 \%)$ & $21(66 \%)$ \\
\hline$-G 1$ & $14(39 \%)$ & $6(19 \%)$ \\
\hline$-G 2$ & $6(17 \%)$ & $3(9.4 \%)$ \\
\hline$-\mathrm{G3}$ & $4(11 \%)$ & $2(6.3 \%)$ \\
\hline$-\mathrm{G} 4$ & $0(0 \%)$ & $0(0 \%)$ \\
\hline \multicolumn{3}{|l|}{ UFT dose per body surface area $\left(\mathrm{mg} / \mathrm{m}^{2}\right)$ : } \\
\hline - UFT plus endocrine therapy & $15(42 \%)$ & $17(53 \%)$ \\
\hline
\end{tabular}

The incidence of grade 1-3 hepatic dysfunction is higher in step 1 than in step 2. No significant differences in age, clinical stage, subtype, UFT dose per body surface area or medication are observed between patients analyzed in step $1(n=36)$ and those analyzed in step $2(n=32)$.

ALT: Alanine transaminase; AST: Aspartate aminotransferase; HR: Hormone receptor; SD: Standard deviation; UFT: Tegafur-uracil.

Odds ratios (OR) were calculated using logistic regression.

An association analysis was also performed in the first and second steps between age, clinical stage, UFT dose per body surface area, medication (UFT alone or plus endocrine therapy), and toxicity using the Jonckheere-Terpstra trend test and between subtype and toxicity using Fisher's exact test. Moreover, an association analysis was performed between genotypes and the amount of change of AST and ALT levels before and after administration of UFT.

Statistical analyses were performed using SAS version 9.1 (SAS Institute Inc., NC, USA). A p-value less than 0.05 was considered significant.

\section{Results}

\section{Patients' characteristics}

The patients' characteristics are shown in Table 2. The incidence of grades $1-3$ hepatic dysfunction was higher in step 1 patients $(\mathrm{n}=36)$ than in step 2 patients $(\mathrm{n}=32)$. However, no significant differences in age, clinical stage, subtype, UFT dose per body surface area or medication were observed between step 1 patients and step 2 patients. 


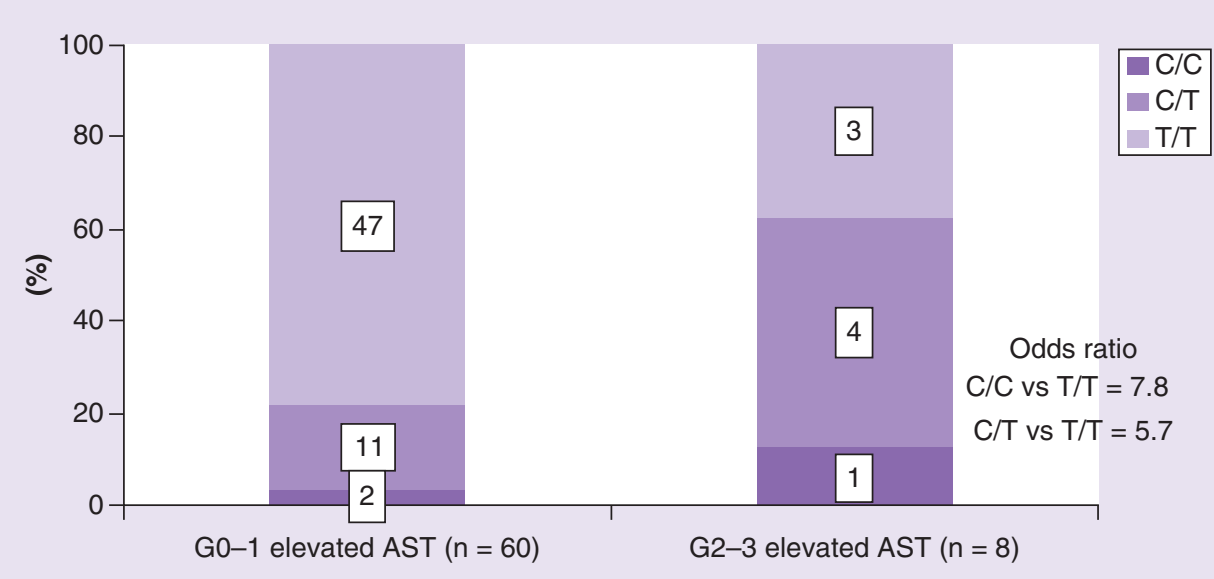

Figure 1. Associations between SLCO1B1 genotypes and G0-3 elevated aspartate aminotransferase levels. The C/C and $C / T$ genotypes are associated with a higher risk of toxicity compared with the $\mathrm{T} / \mathrm{T}$ genotype (odds ratio: $\mathrm{C} / \mathrm{C}$ vs $\mathrm{T} / \mathrm{T}=7.8, \mathrm{C} / \mathrm{T}$ vs $\mathrm{T} / \mathrm{T}=5.7$ ) in 68 patients from steps 1 and 2 . A total of 47 of 60 patients have the $\mathrm{T} / \mathrm{T}$ genotype, 11 have the $\mathrm{C} / \mathrm{T}$ genotype and two have the $\mathrm{C} / \mathrm{C}$ genotype in the $\mathrm{G} 0-1$ group. Three of eight patients have the $\mathrm{T} / \mathrm{T}$ genotype, four have the C/T genotype and one has the C/C genotype in the G2-3 group.

Additionally, there were no associations between age, clinical stage, subtype, UFT dose per body surface area or medication and elevated AST and ALT levels in both steps 1 and 2.

\section{Associations between genotypes \& elevated AST \& ALT levels in step 1, step 2 \& both steps 1 \& 2}

Since it was not possible to estimate a suitable genetic model, three genetic models (additive, recessive and dominant) were examined for the association study. Table 3 shows the 20 SNPs in 17 genes that showed $p<0.05$ in at least one of the three genetic models for both AST and ALT.

Then, based on previous clinical trials in Japan [7], the frequency of UFT-induced elevated AST and ALT levels was estimated at approximately 15-25\%; the SNPs whose frequency of risk allele was 15-25\% were selected. Thus, among the 20 SNPs, five (AOX1, CYP24A1, SLC7A7, SLC22A1 and SLCO1B1) were finally selected, taking into account both the risk allele frequency of these SNPs in the Japanese population and the frequency of UFT-induced elevated AST and ALT levels. Tables 4 and 5 show the results of first-step and second-step analysis of the associations between the five candidate SNPs and elevated AST and ALT levels and the risk alleles for elevated AST and ALT levels. Only SLCO1B1 was significantly associated with both elevated AST $(\mathrm{p}=0.037)$ and ALT $(\mathrm{p}=0.034)$ levels with the C/C genotype among the candidate SNPs. In step $1+2$, focusing on the change in the percentage of grade 2-3 elevated AST levels for every genotype in SLCO1B1, there was a tendency for the frequency of elevated AST levels to increase with many minor alleles: $6.0 \%$ for $\mathrm{T} / \mathrm{T}, 26.7 \%$ for $\mathrm{C} / \mathrm{T}$ and $33.3 \%$ for $\mathrm{C} / \mathrm{C}$. On the other hand, this tendency was not seen with other candidate genes. The ALT levels showed a tendency to increase that was similar to the AST levels.

Only one SNP, 521T > C (rs4149056) of SLCO1B1, showed a significant association with UFT treatment-related elevation of both AST and ALT levels. The 68 patients examined in step 1 or 2 were then categorized into two groups, grades $0-1$ and grades $2-3$ elevated AST and ALT levels, and the genotype frequencies and OR were determined (Figures $1 \& 2$ ). The $\mathrm{C} / \mathrm{C}$ genotype was associated with a higher risk of hepatic dysfunction compared with the $\mathrm{T} / \mathrm{T}$ genotype (AST OR $=7.8$; ALT OR $=12.2$ ). The $\mathrm{C} / \mathrm{T}$ genotype was also associated with a higher risk of hepatic dysfunction than the $\mathrm{T} / \mathrm{T}$ genotype (AST $\mathrm{OR}=5.7$; ALT OR $=4.1$ ). These results suggest that individuals with $\mathrm{C} / \mathrm{C}$ and $\mathrm{C} / \mathrm{T}$ genotypes have a greater risk of UFT-induced hepatic dysfunction. On unified analysis of the first and second steps, the results showed $\mathrm{p}<0.05$ in the two genetic models for AST and in the three genetic models for ALT.

The predicted occurrence of grades 2-3 elevated AST and ALT levels according to genotype frequency were 54 and $67 \%$ for $\mathrm{C} / \mathrm{C}, 46$ and $40 \%$ for $\mathrm{C} / \mathrm{T}$, and 13 and $14 \%$ for $\mathrm{T} / \mathrm{T}$, respectively. The predicted occurrence was calculated based on the following: the assumption that the frequency of UFT-induced elevated AST and ALT levels was $20 \%$ in the Japanese population; data from the International HapMap Project [16] on the genotype frequencies 


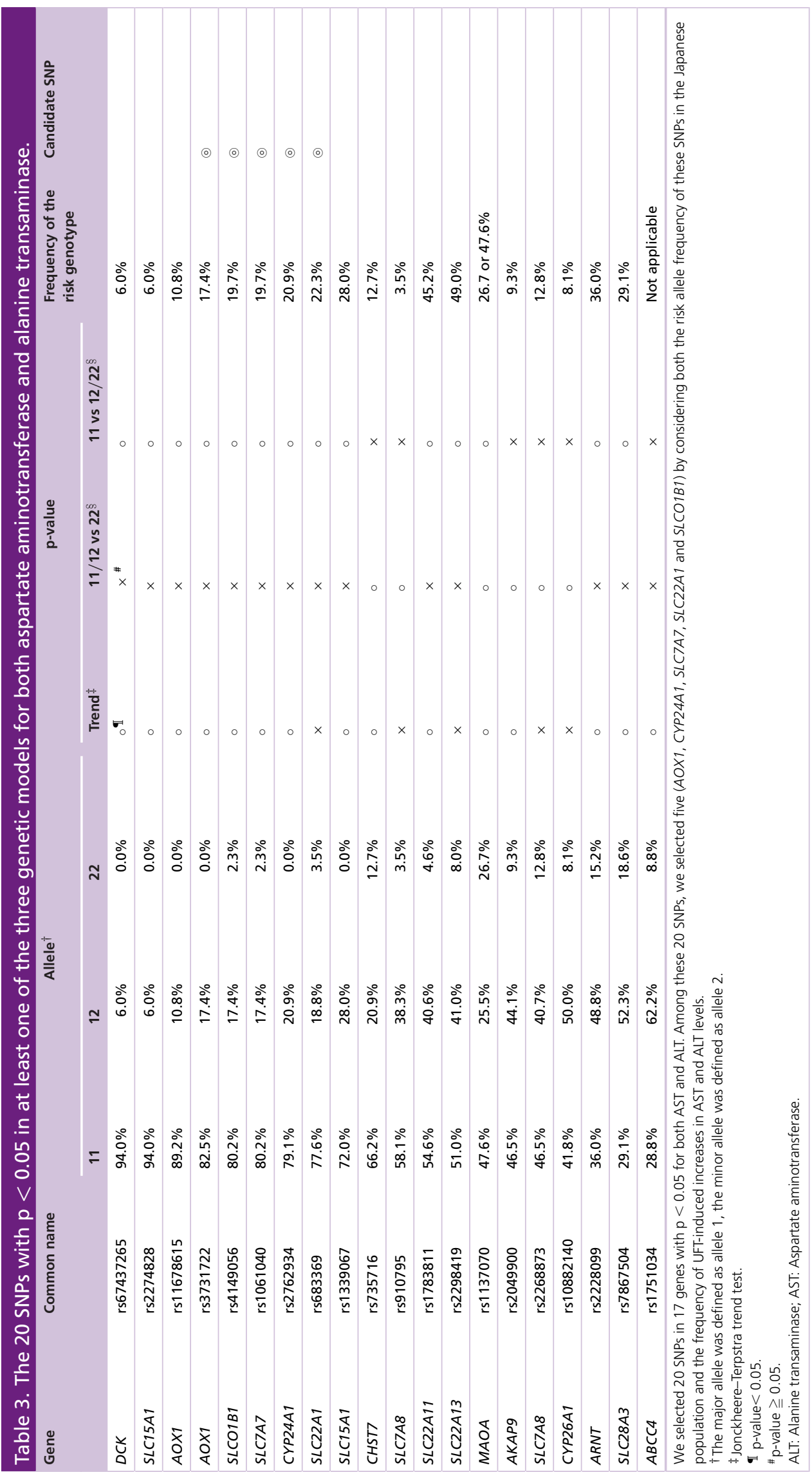




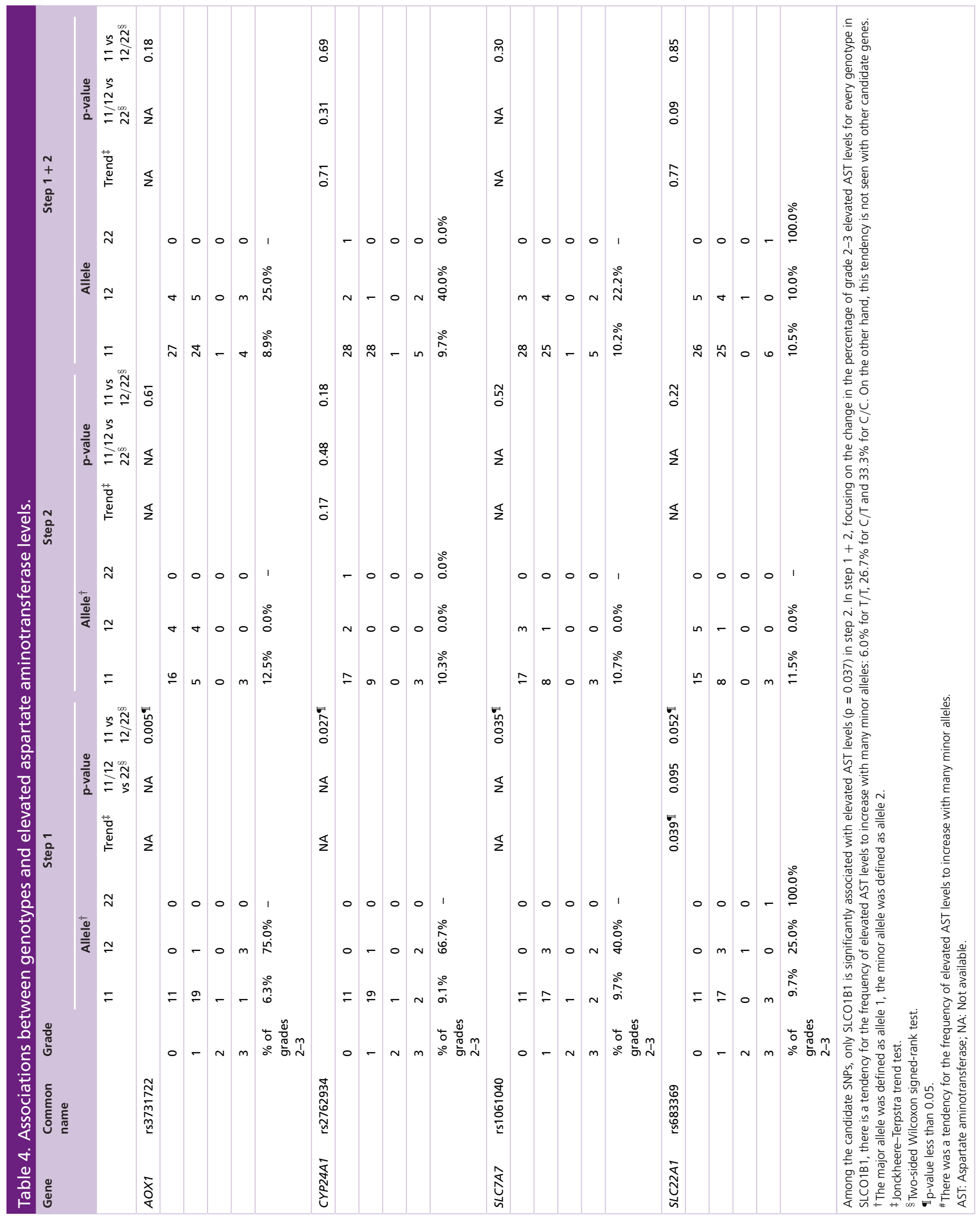




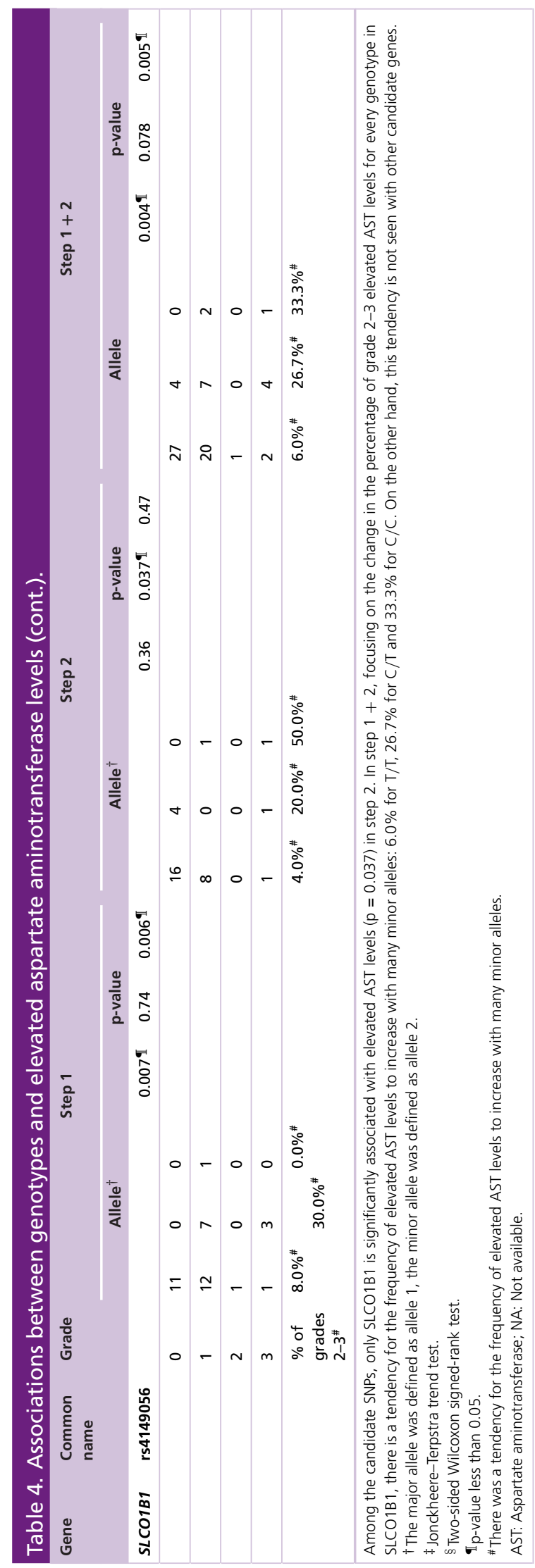




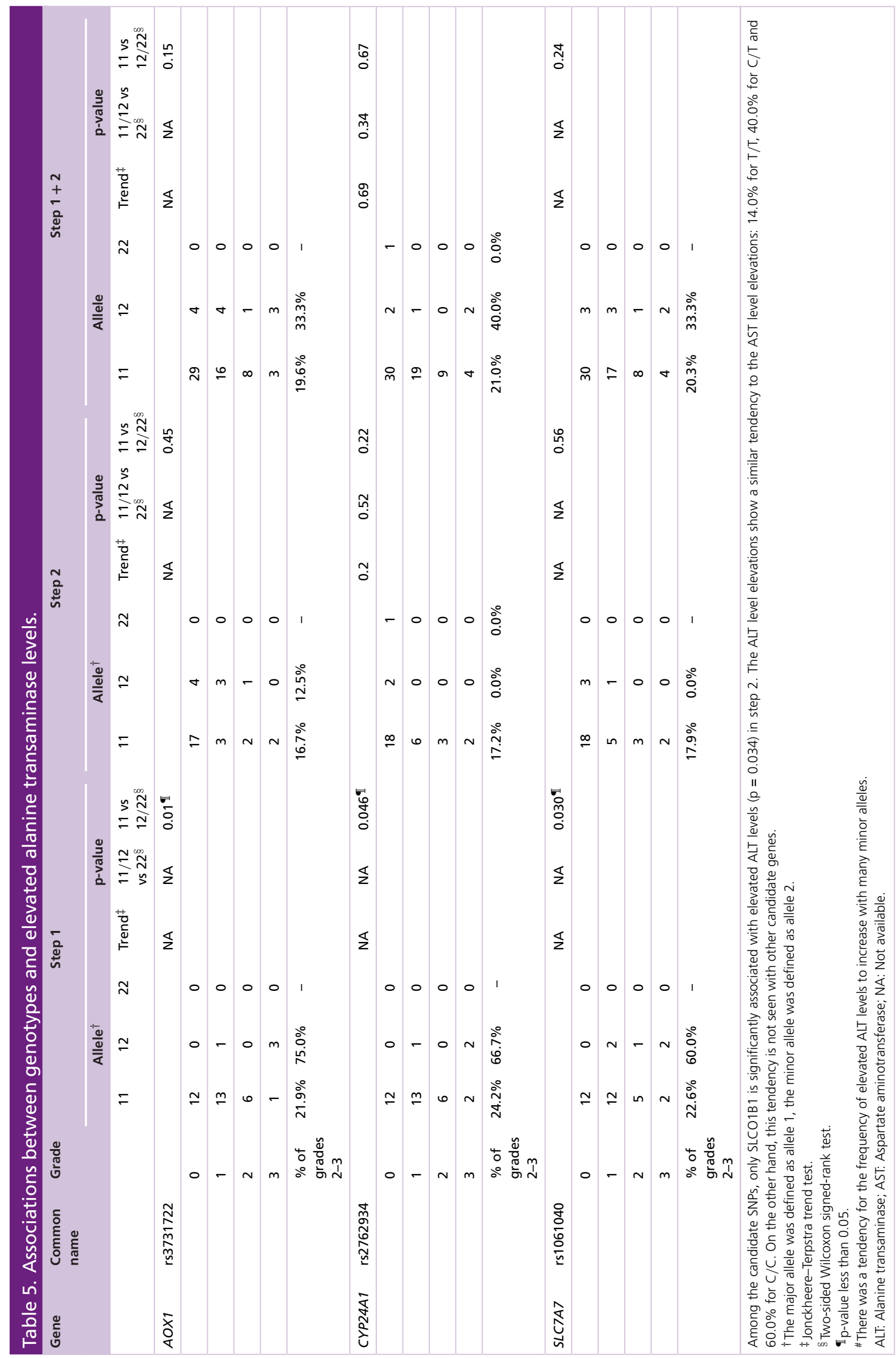




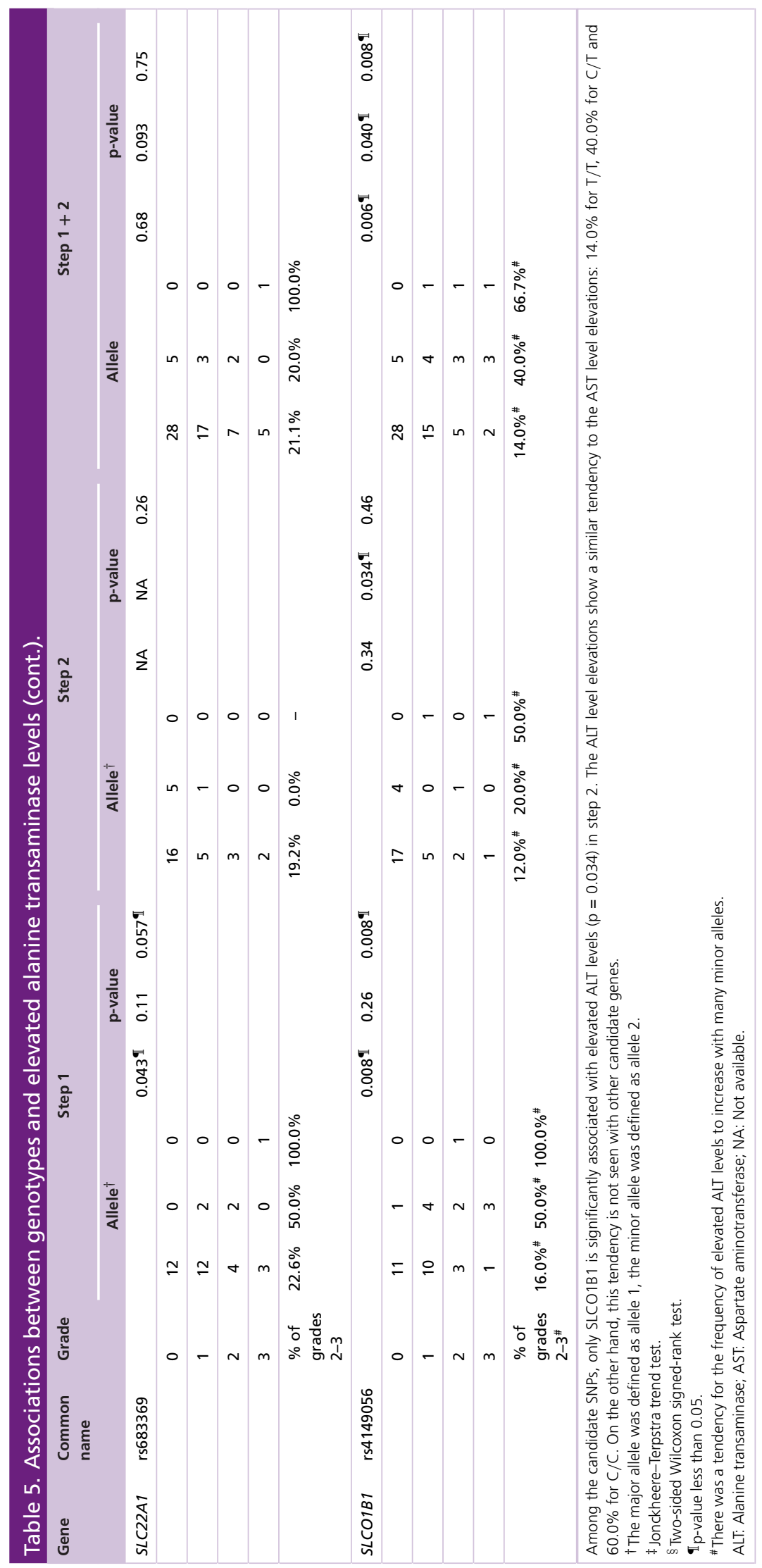




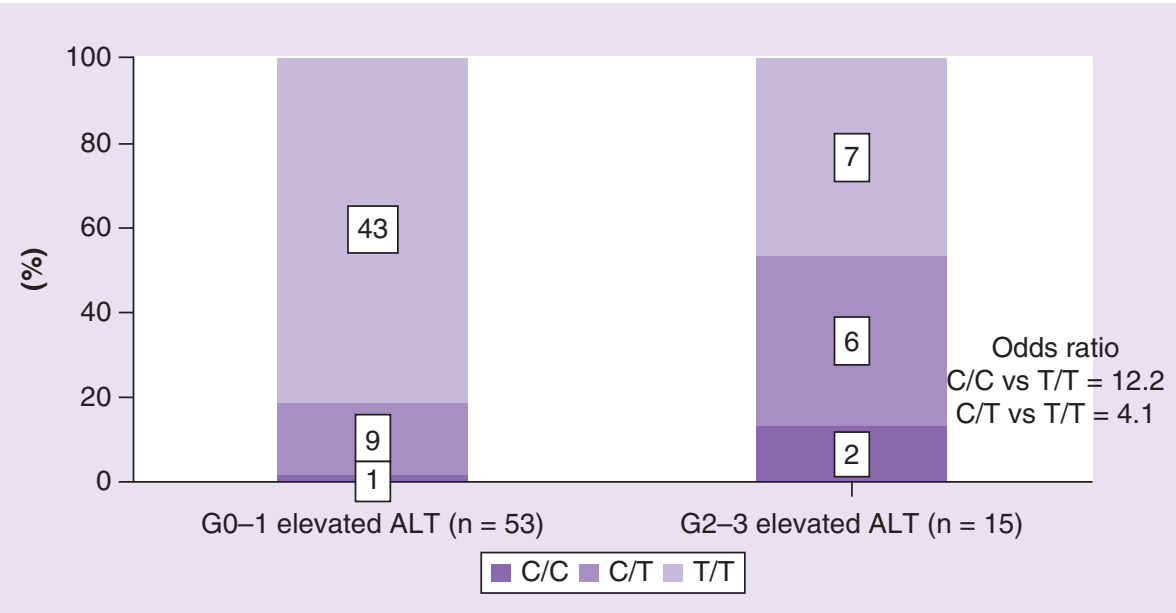

Figure 2. Associations between SLCO1B1 genotypes and G0-3 elevated alanine transaminase levels. The C/C and $\mathrm{C} / \mathrm{T}$ genotypes are associated with a higher risk of toxicity compared with the $\mathrm{T} / \mathrm{T}$ genotype (odds ratio: $\mathrm{C} / \mathrm{C}$ vs $\mathrm{T} / \mathrm{T}=12.2, \mathrm{C} / \mathrm{T}$ vs $\mathrm{T} / \mathrm{T}=4.1$ ) in 68 patients from steps 1 and 2 . A total of 43 of 53 patients have the $\mathrm{T} / \mathrm{T}$ genotype, nine have the $\mathrm{C} / \mathrm{T}$ genotype and one has the $\mathrm{C} / \mathrm{C}$ genotype in the $\mathrm{GO}-1$ group. Seven of 15 patients have the T/T genotype, six have the $\mathrm{C} / \mathrm{T}$ genotype and two have the $\mathrm{C} / \mathrm{C}$ genotype in the $\mathrm{G} 2-3$ group.

of SLCO1B1 rs4149056 in the Japanese population ( $\mathrm{T} / \mathrm{T}=0.802, \mathrm{C} / \mathrm{T}=0.174$ and $\mathrm{C} / \mathrm{C}=0.023)$; and the $\mathrm{OR}$ calculated in the present study.

The results of an association analysis between genotypes in SLCO1B1 and the increment of AST values before and after administration of UFT showed median values of 44 for $\mathrm{C} / \mathrm{C}, 27.5$ for $\mathrm{C} / \mathrm{T}$ and 6.0 for $\mathrm{T} / \mathrm{T}$ in the first step and 156.5 for $\mathrm{C} / \mathrm{C}, 9.0$ for $\mathrm{C} / \mathrm{T}$ and 6.0 for $\mathrm{T} / \mathrm{T}$ in the second step. The results of step 1 and step 2 showed a similar tendency, and the results for ALT showed a tendency similar to those for AST.

Overall, these results suggest that individuals with $\mathrm{C} / \mathrm{C}$ and $\mathrm{C} / \mathrm{T}$ genotypes have a greater risk of UFT-induced hepatic dysfunction.

\section{Discussion}

In the present study, the associations between SNPs in most drug-metabolizing enzymes and drug transporters and UFT-induced hepatic dysfunction were investigated in breast cancer patients treated with UFT. The findings showed that SLCO1B1 rs4149056 was associated with UFT-induced hepatic dysfunction.

Several studies have attempted to elucidate the associations between candidate genes and 5-FU-induced toxicities; polymorphisms in DPYD and TYMS were reportedly associated with 5-FU-induced severe toxicities such as diarrhea, mucositis and leucopenia [10,16-18]. Conversely, other reports demonstrated that DPYD and TYMS polymorphisms failed to predict 5-FU-induced toxicities [11-13]. A recent study [19] using the DMET genotyping system showed that both CHST1 and GSTM3 were associated with 5-FU-induced gastrointestinal toxicity in colorectal cancer patients. In the present study, none of these SNPs was associated with UFT-induced hepatic dysfunction. It has also been reported that there is no significant association between SLCO1B1 rs2306283 and 5-FU+irinotecan-induced toxicities such as diarrhea and mucositis in rectal cancer patients [20]. However, this study was different from the present study with respect to target cancer, target race, target side effect, chemotherapy regimen and polymorphism in SLCO1B1.

According to the findings of a clinical trial of Japanese breast cancer patients administered UFT, approximately $20 \%$ of patients developed grades 1-4 elevated AST and ALT levels [7,8]. According to data from the International HapMap Project [16], in the Japanese population, the combined genotype frequency of C/C ( = 0.023) and C/T ( = 0.174) in SLCO1B1 rs 4149056 is $19.7 \%$. This suggests that the genotype frequencies of C/C and C/T are generally consistent with the occurrence of grades $1-4$ hepatic dysfunction observed during clinical trials.

In our study, SLCO1B1 rs4149056 was associated with not only grade 2-3 elevated AST and ALT but also grade 1-3 elevated AST and ALT. Then SLCO1B1 rs4149056 may become the useful biomarker for the liver dysfunction more than grade 1 . Herein, we wrote the results of regarding only the comparison between grade $0-1$ and grade 
2-3 because liver dysfunction more than grade 2 needs clinical intervention. But we need the attention regarding grade 1 elevated aminotransferase, because it may develop into grade 2.

The SLCO1B1 gene encodes for a membrane-bound sodium-independent OATP1B1. The OATP1B1 is predominantly expressed on the basolateral membrane of hepatocytes and can facilitate hepatic uptake of certain clinically relevant drugs such as statins, angiotensin-converting enzyme inhibitors, angiotensin II receptor antagonists, an antidiabetic drug and anticancer drugs [21-23]. In the present study, no patient was administered these drugs while taking UFT. Previous studies have not reported an association between SLCO1B1 and 5-FU pharmacokinetics and pharmacodynamics.

The SNP rs4149056 is nonsynonymous and has been shown to decrease drug uptake, leading to increased plasma concentrations [24-27]. Although it has not been shown that OATP1B1 transports 5-FU, we hypothesize that rs4149056 causes a functional abnormality of OATP1B1, leading to hepatic dysfunction with UFT.

The limitation of the present study was the small number of cases. Furthermore, hepatic dysfunction is a multifactorial phenomenon depending on several factors. Although there were no associations between nongenetic factors such as age, clinical stage, subtype, UFT dose per body surface area or medication and elevated AST and ALT levels in the present study, a study of a large population is needed to confirm the results of the present study and to elucidate the physiological significance of this SNP.

\section{Conclusion}

The SNP rs4149056 in SLCO1B1 was associated with UFT treatment-related elevations of AST (OR: C/C vs $\mathrm{T} / \mathrm{T}=7.8, \mathrm{C} / \mathrm{T}$ vs $\mathrm{T} / \mathrm{T}=5.7$ ) and elevations of ALT (OR: $\mathrm{C} / \mathrm{C}$ vs $\mathrm{T} / \mathrm{T}=12.2, \mathrm{C} / \mathrm{T}$ vs $\mathrm{T} / \mathrm{T}=4.1$ ) levels. It showed a significant association with them in both the first and second-step analyses. It also showed a significant association with them in relation to both the grade and the increments of AST and ALT values.

In conclusion, the present findings suggest that $S L C O 1 B 1$ polymorphisms can potentially predict UFT-induced hepatic dysfunction.

\section{Summary points}

- Hepatic dysfunction is a serious adverse effect of tegafur-uracil (UFT) therapy, affecting approximately $20 \%$ of patients.

- The aim of this study was to identify pharmacogenomic biomarkers for the prediction of UFT-induced liver dysfunction.

- In the first step, 36 patients were analyzed using the DMET ${ }^{\mathrm{TM}}$ Plus GeneChip microarray and we observed an association between five SNPs and UFT-induced hepatic dysfunction.

- In the second step, the remaining 32 patients were analyzed using DNA sequencing and an SNP in SLCO1B1 was significantly associated with both elevated AST and ALT levels among the candidate SNPs.

- The present findings suggest that SLCO1B1 polymorphisms can potentially predict UFT-induced hepatic dysfunction.

\section{Acknowledgements}

The authors would like to thank all of the study participants who provided tissue samples for this analysis. They are also indebted to T Aoki for her excellent technical assistance.

Financial \& competing interests disclosure

The authors have no relevant affiliations or financial involvement with any organization or entity with a financial interest in or financial conflict with the subject matter or materials discussed in the manuscript. This includes employment, consultancies, honoraria, stock ownership or options, expert testimony, grants or patents received or pending, or royalties.

No writing assistance was utilized in the production of this manuscript.

\section{Ethical disclosure}

The study was approved by the Institutional Review Board of Tokyo Women's Medical University (approval no. 248) and was in compliance with the Declaration of Helsinki. Written, informed consent was obtained from all individuals that participated in the study. 


\section{References}

Papers of special note have been highlighted as: $\bullet$ of interest; $\bullet \bullet$ of considerable interest

1. Nakajima T, Kinoshita T, Nashimoto A et al. Randomized controlled trial of adjuvant uracil-tegafur versus surgery alone for serosa-negative, locally advanced gastric cancer. Br. J. Surg. 94, 1468-1476 (2007).

2. Akasu T, Moriya Y, Ohashi Y, Yoshida S, Shirao K, Kodaira S. Adjuvant chemotherapy with uracil-tegafur for pathological stage III rectal cancer after mesorectal excision with selective lateral pelvic lymphadenectomy: a multicenter randomized controlled trial. Jpn J. Clin. Oncol. 36, 237-244 (2006).

3. Kato $\mathrm{H}$, Ichinose $\mathrm{Y}$, Ohta $\mathrm{M}$ et al. A randomized trial of adjuvant chemotherapy with uracil-tegafur for adenocarcinoma of the lung. $N$. Engl. J. Med. 350, 1713-1721 (2005).

4. Goldhirsch A, Wood WC, Coates AS, Gelber RD, Thürlimann B, Senn HJ. Strategies for subtypes - dealing with the diversity of breast cancer: highlights of the St. Gallen International Expert Consensus on the Primary Therapy of Early Breast Cancer 2011. Ann. Oncol. 22, 1736-1747 (2011).

5. Ohashi Y, Watanabe T, Sano M, Koyama H, Inaji H, Suzuki T. Efficacy of oral tegafur-uracil (UFT) as adjuvant therapy as compared with classical cyclophosphamide, methotrexate, and 5-fluorouracil (CMF) in early breast cancer: a pooled analysis of two randomized controlled trials (N-SAS-BC 01 trial and CUBC trial). Breast Cancer Res. Treat. 119, 633-641 (2010).

6. Watanabe T. Evidence produced in Japan: tegafur-based preparations for postoperative chemotherapy in breast cancer. Breast Cancer 20, 302-309 (2013).

7. Watanabe T, Sano M, Takashima $S$ et al. Oral uracil and tegafur compared with classic cyclophosphamide, methotrexate, fluorouracil as postoperative chemotherapy in patients with node-negative, high-risk breast cancer: National Surgical Adjuvant Study for Breast Cancer 01 Trial. J. Clin. Oncol. 27, 1368-1374 (2009).

\section{- A clinical trial that evaluated the side effects of tegafur-uracil.}

8. Taguchi T, Noguchi S. [Safety and compliance with UFT (tegafur and uracil) alone and in combination with hormone therapy in patients with breast cancer]. Jpn J. Cancer Chemother. 36, 1465-1474 (2009).

9. Ohmoto K, Ohashi K, Iguchi Y et al. [A case of drug-induced fulminant hepatitis]. Clin. Gastroenterol. 10, 705-709 (1995).

10. Rosmarin D, Palles C, Church D et al. Genetic markers of toxicity from capecitabine and other fluorouracil-based regimens: investigation in the QUASAR2 study, systematic review, and meta-analysis. J. Clin. Oncol. 32, 1031-1039 (2014).

-• A meta-analysis that assessed the associations between toxicities of 5-FU and many genetic polymorphisms associated with FU metabolism.

11. Schwab M, Zanger UM, Marx C et al. Role of genetic and nongenetic factors for fluorouracil treatment-related severe toxicity: a prospective clinical trial by the German 5-FU Toxicity Study Group. J. Clin. Oncol. 26, 2131-2138 (2008).

12. Gusella M, Frigo AC, Bolzonella $\mathrm{C}$ et al. Predictors of survival and toxicity in patients on adjuvant therapy with 5-fluorouracil for colorectal cancer. Br. J. Cancer 100, 1549-1557 (2009).

13. Ghosh $\mathrm{S}$, Hossain MZ, Borges $\mathrm{M}$ et al. Analysis of polymorphisms and haplotype structure of the human thymidylate synthase genetic region: a tool for pharmacogenetic studies. PLoS ONE 7, e34426 (2012).

14. Marsh S. Impact of pharmacogenomics on clinical practice in oncology. Mol. Diagn. Ther. 11, 79-82 (2007).

15. The International HapMap Consortium. The International HapMap Project. Nature 426, 789-796 (2003).

16. Gentile G, Botticelli A, Lionetto L et al. Genotype-phenotype correlations in 5-fluorouracil metabolism: a candidate DPYD haplotype to improve toxicity prediction. Pharmacogenomics J. 16, 320-325 (2016).

17. Afzal S, Gusella M, Vainer B et al. Combinations of polymorphisms in genes involved in the 5-fluorouracil metabolism pathway are associated with gastrointestinal toxicity in chemotherapy-treated colorectal cancer patients. Clin. Cancer Res. 17, 3822-3829 (2011).

18. Lecomte T, Ferraz JM, Zinzindohoué $\mathrm{F}$ et al. Thymidylate synthase gene polymorphism predicts toxicity in colorectal cancer patients receiving 5-fluorouracil-based chemotherapy. Clin. Cancer Res. 10, 5880-5888 (2004).

19. Rumiato E, Boldrin E, Amadori A, Saggioro D. DMET ${ }^{T M}$ (drug-metabolizing enzymes and transporters) microarray analysis of colorectal cancer patients with 5-fluorouracil-induced toxicity. Cancer Chemother. Pharmacol. 72, 483-488 (2013).

20. Thomas F, Motsinger-Reif AA, Hoskins JM et al. Methylenetetrahydrofolate reductase genetic polymorphisms and toxicity to 5-FU based chemoradiation in rectal cancer. Br. J. Cancer 105, 1654-1662 (2011).

21. König J, Cui Y, Nies AT, Keppler D. A novel human organic anion transporting polypeptide localized to the basolateral hepatocyte membrane. Am. J. Physiol. Gastrointest. Liver Physiol. 278, 156-164 (2000).

22. Hsiang B, Zhu Y, Wang Z et al. A novel human hepatic organic anion transporting polypeptide (OATP2). Identification of a liver-specific human organic anion transporting polypeptide and identification of rat and human hydoxymethylglutary1-CoA reductase inhibitor transporters. J. Biol. Chem. 274, 37161-37168 (1999).

23. Ieiri I, Higuchi S, Sugiyama Y. Genetic polymorphisms of uptake (OPTP1B1, 1B3) and efflux (MRP2, BCRP) transporters: implications for inter-individual differences in the pharmacokinetics and pharmacodynamics of statins and other clinically relevant drugs. Expert Opin. Drug Metab. Toxicol. 5, 1-27 (2009). 
24. Tirona RG, Leake BF, Merino G, Kim RB. Polymorphisms in OATP-C: identification of multiple allelic variants associated with altered transport activity among European- and African-Americans. J. Biol. Chem. 276, 35669-35675 (2001).

25. Ho RH, Tirona RG, Leake BF et al. Drug and bile acid transporters in rosuvastatin hepatic uptake: function, expression, and pharmacogenetics. Gastroenterology 130, 1793-1806 (2006).

26. Iwai M, Suzuki H, Ieiri I, Otsubo K, Sugiyama Y. Functional analysis of single nucleotide polymorphisms of hepatic organic anion transporter OATP1B1 (OATP-C). Pharmacogenetics 14, 749-757 (2004).

27. Kameyama Y, Yamashita K, Kobayashi K, Hosokawa M, Chiba K. Functional characterization of SLCO1B1 (OATP-C) variants, SLCO1B1*5, SLCO1B1*15 and SLCO1B1*15+C1007G, by using transient expression systems of HeLa and HEK293 cells. Pharmacogenet. Genomics 15, 513-522 (2005).

-• One of the most important pharmacogenetics studies assessing the impact of SLCO1B1 polymorphisms. 
